\title{
Evaluating the effectiveness of IPTi on malaria using routine health information from sentinel health centres in southern Tanzania
}

\author{
Barbara A Willey ${ }^{1}$, Joanna RM Armstrong Schellenberg', Werner Maokola², Kizito Shirima², Mwajuma Chemba², \\ Hassan Mshinda ${ }^{2}$, Pedro Alonso ${ }^{3}$, Marcel Tanner ${ }^{4,5}$, David Schellenberg ${ }^{2 *}$
}

\begin{abstract}
Background: Intermittent preventive treatment of malaria in infants (IPTi) consists of the administration of a treatment dose of sulphadoxine-pyrimethamine (SP) at the time of routine vaccinations. The use of routine Health Management and Information Services (HMIS) data to investigate the effect of IPTi on malaria, anaemia, and allcause attendance in children aged 2-11 months presenting to 11 health centres in southern Tanzania is described.

Methods: Clinical diagnosis of malaria was confirmed with a positive blood slide reading from a quality assurance laboratory. Anaemia was defined using two thresholds (mild $[\mathrm{Hb}<11 \mathrm{~g} / \mathrm{dL}]$, severe $[\mathrm{Hb}<8 \mathrm{~g} / \mathrm{dL}]$ ). Incidence rates between IPTi and non-implementing health centres were calculated using Poisson regression, and all statistical testing was based on the t test due to the clustered nature of the data.

Results: Seventy two per cent of infants presenting in intervention areas received at least one dose of IPTi- 22\% received all three. During March 2006 - April 2007, the incidence of all cause attendance was two attendances per person, per year (pppy), including 0.2 episodes pppy of malaria, 0.7 episodes of mild and 0.13 episodes of severe anaemia. Point estimates for the effect of IPTi on malaria varied between $18 \%$ and $52 \%$, depending on the scope of the analysis, although adjustment for clustering rendered these not statistically significant.

Conclusions: The point estimate of the effect of IPTi on malaria is consistent with that from a large pooled analysis of randomized control trials. As such, it is plausible that the difference seen in health centre data is due to IPTi, even thought the effect did not reach statistical significance. Findings draw attention to the challenges of robust inference of effects of interventions based on routine health centre data. Analysis of routine health information can reassure that interventions are being made available and having desired effects, but unanticipated effects should trigger data collection from representative samples of the target population.
\end{abstract}

\section{Background}

The contribution of Plasmodium falciparum-caused malaria to mortality and morbidity of African infants and young children remains substantial [1]. Malaria control strategies throughout the African continent have seen considerable increases in financial and human resources investment from governments, non-governmental agencies, and funders alike [1]. The monitoring and evaluation of malaria control programmes is essential, and recently more emphasis has been placed on both by the

\footnotetext{
* Correspondence: david.schellenberg@lshtm.ac.uk

If fakara Health Institute, Dar es Salaam, Tanzania

Full list of author information is available at the end of the article
}

WHO's Global Malaria Programme and by the Roll Back Malaria (RBM) partnership. RBM's Monitoring and Evaluation Reference Group (MERG) recommends a number of data collection methods to monitor and evaluate malaria control strategies, and highlights as currently under-exploited, the use of routine health management and information system, particularly from randomlyselected malaria sentinel sites [2,3].

Intermittent preventive treatment of malaria in infants (IPTi) consists of the administration of a treatment dose of sulphadoxine-pyrimethamine (SP) at the time of routine vaccinations in the first year of life [4]. In a pooled analysis of six randomized controlled trials (RCTs) of

\section{() Biomed Central}

(C) 2011 Willey et al; licensee BioMed Central Ltd. This is an Open Access article distributed under the terms of the Creative Commons Attribution License (http://creativecommons.org/licenses/by/2.0), which permits unrestricted use, distribution, and reproduction in any medium, provided the original work is properly cited. 
IPTi using SP, the incidence of clinical malaria in the first year of life was reduced by $30 \%$ [5], and IPTi was considered a safe and effective anti-malarial strategy, likely to form a useful component of malaria control programmes in relevant settings [6]. In addition to RCTs [5,7-12], studies addressing questions of cost $[13,14]$, safety, drug resistance [15], acceptability [16,17], and the implementation of IPTi through the Expanded Programme on Immunization (EPI) [18] have also been carried out, under the umbrella of the IPTi consortium [19]. The community effectiveness of IPTi and its impact on infant health has been investigated in Tanzania through a cluster-randomized controlled trial including representative pre and post-intervention household surveys $[20,21]$.

In this paper, data from a consolidated HMIS in 11 sentinel health centres in rural southern Tanzania are used to report (i) the uptake of IPTi and coverage of corresponding EPI vaccines, (ii) the incidence of malaria, anaemia, and all-cause attendance at the health centre, and (iii) the effectiveness of IPTi on the incidence of these outcomes in health centre attendees aged 2-11 months.

\section{Methods}

\section{Study area and the IPTi strategy}

The area has been previously described, but briefly malaria is endemic, with transmission occurring throughout the year, and is the most common primary cause of health centre or hospital admission among those aged under five years [20,22]. The first-line antimalarial drug in this area was SP, until December 2006, when it changed to artemether-lumefantrine. During the study period a number of malaria control interventions were implemented. Nationwide, from May 2006, the Tanzania National Voucher Scheme was in effect, delivering bed nets bundled with insecticide re-treatment kits to pregnant women through vouchers available at antenatal care clinics [23]. Health centres nationwide also distributed free insecticide re-treatment kits to infants when they attended government clinics for measles vaccination. Furthermore, in Lindi region (Figure 1) during August 2005, bed nets bundled with insecticide were distributed to children aged less than five years during an integrated mass measles vaccination, helminth treatment, and vitamin A supplementation campaign [24].

This study, termed the IPTi Morbidity Monitoring study, took place in the context of a large cluster randomized trial that was designed to determine the community effectiveness of IPTi with SP on malaria and anaemia. IPTi using SP was introduced to 12 of 24 randomly selected divisions, located within five districts, and two regions (Lindi and Mtwara) of rural southern

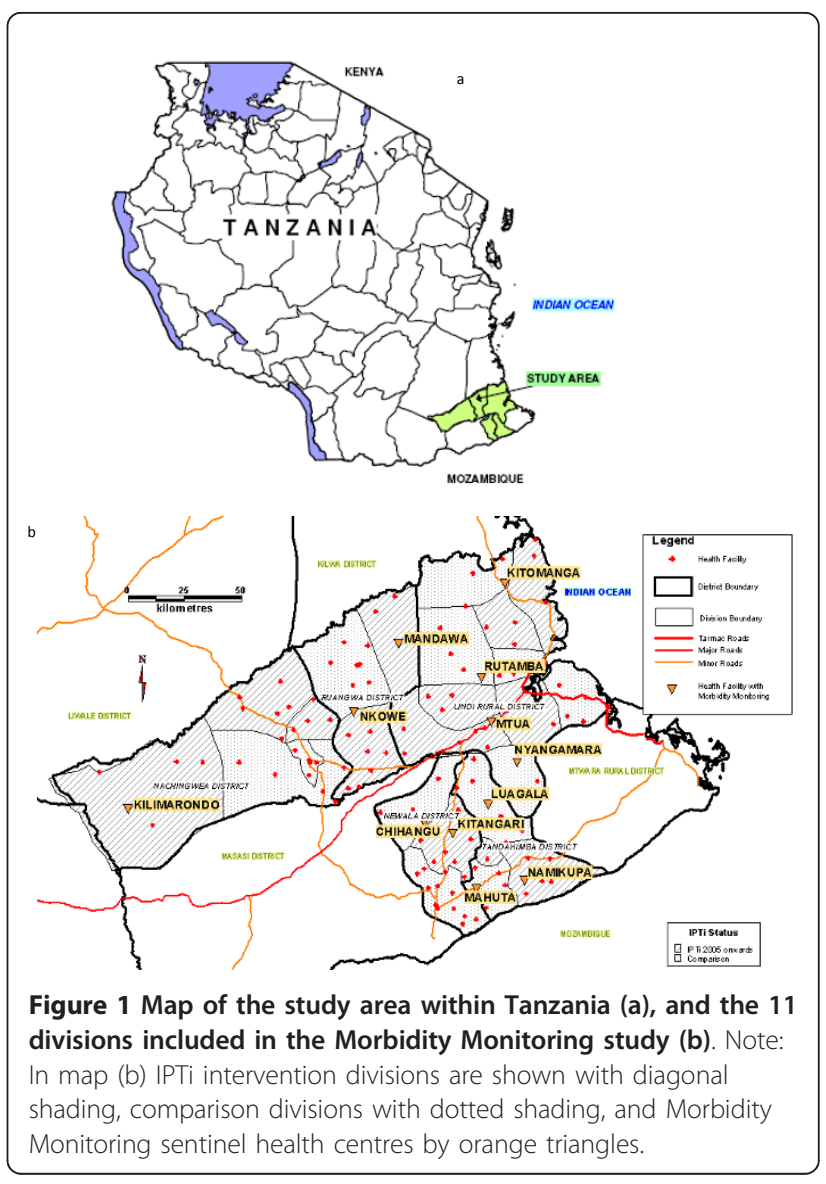

Tanzania (Figure 1) [22]. IPTi effectiveness was estimated through representative household surveys that took place in all 24 participating divisions at baseline in $2004(\mathrm{n}=21,600)$, and post IPTi in July/August 2006 $(\mathrm{n}=5,760)$ [20]. IPTi, in the form of a single dose of $\mathrm{SP}$, was delivered in intervention divisions through existing government health centres when children presented for their routine EPI vaccine doses of DPT2, DPT3, and measles (given at two, three, and nine months of age, respectively). Children presenting at government health centres in comparison divisions received their routine EPI vaccine, but no IPTi.

\section{The IPTi Morbidity Monitoring study, data collection and} outcome definition

The Morbidity Monitoring study aimed to monitor the delivery of IPTi with SP through routine contacts with the health service, and to enable evaluation of the effectiveness of IPTi using routine health centre data. All 11 health centres with functional microscopes in the study area were included. These were located in 11 of the 24 divisions included in the IPTi trial, six in intervention, and five in comparison areas (Figure 1, Table 1). On the basis of routine data available for 2004, intervention and comparison 
Table 1 Distribution of children by health centre, characteristics of the Morbidity Monitoring study population, and uptake of EPI and IPTi doses

\begin{tabular}{lll}
\hline & Intervention & Comparison \\
\hline Number children per health centre $[\mathrm{n},(\%)]$ & $\mathrm{n}=5035$ & $\mathrm{n}=4845$ \\
$\mathrm{~A}$ & $486(4.9)$ & \\
B & $869(8.9)$ & \\
C & $862(8.7)$ & \\
D & $999(10.1)$ & \\
E & $951(9.6)$ & \\
F & $868(8.8)$ & $840(8.5)$ \\
G & & $519(5.2)$ \\
H & & $1198(12.1)$ \\
I & & $1610(16.3)$ \\
J & & $678(6.9)$ \\
K & &
\end{tabular}

Sex $[n / N,(\%)]$

\begin{tabular}{|c|c|c|c|}
\hline Male & $2567 / 5035(51)$ & $2514 / 4845(52)$ & 0.525 \\
\hline \multicolumn{4}{|l|}{ Birth order $[\mathrm{n} / \mathrm{N},(\%)]$} \\
\hline Singleton & 4912/5035(98) & $4685 / 4845(97)$ & 0.533 \\
\hline Median age at entry into analysis [months, (IQR)] & $4 \mathrm{~m} 3 \mathrm{~d}(2 \mathrm{~m}-7 \mathrm{~m} 24 \mathrm{~d})$ & 4 m 12 d (2 m-7 m 27 d) & 0.742 \\
\hline \multicolumn{4}{|l|}{ Health centre attendance } \\
\hline$\geq 1$ health centre attendance $[\mathrm{n} / \mathrm{N},(\%)]$ & $2160 / 5035(43)$ & $2067 / 4845(43)$ & 0.799 \\
\hline Median number of attendances (IQR) & $2(1-3)$ & $2(1-3)$ & 0.892 \\
\hline \multicolumn{4}{|l|}{ EPI vaccine uptake $[\mathrm{n} / \mathrm{N},(\%)]$} \\
\hline DPT dose 2 & $3618 / 5035(72)$ & $3181 / 4845(66)$ & 0.204 \\
\hline DPT dose 3 & $2889 / 4794(60)$ & $2296 / 4662(49)$ & 0.019 \\
\hline Measles vaccine & 1609/3208 (50) & 1009/3065 (33) & 0.003 \\
\hline \multicolumn{4}{|l|}{ Median age at EPI vaccinations [months, (IQR)] } \\
\hline DPT dose 2 & $2 \mathrm{~m} 18 \mathrm{~d}(2 \mathrm{~m} 6 \mathrm{~d}-3 \mathrm{~m} 9 \mathrm{~d})$ & 2 m 24 d (2 m 9 d-3 m $21 d)$ & 0.435 \\
\hline DPT dose 3 & $4 \mathrm{~m} 0 \mathrm{~d}(3 \mathrm{~m} 9 \mathrm{~d}-5 \mathrm{~m} 0 \mathrm{~d})$ & $4 \mathrm{~m} 3 \mathrm{~d}(3 \mathrm{~m} 12 \mathrm{~d}-5 \mathrm{~m} 9 \mathrm{~d})$ & 0.585 \\
\hline Measles vaccination & $9 \mathrm{~m} 18 \mathrm{~d}(9 \mathrm{~m} 6 \mathrm{~d}-10 \mathrm{~m} 15 \mathrm{~d})$ & 9 m 15 d (9 m 3 d-10 m6 d) & 0.099 \\
\hline \multicolumn{4}{|l|}{ IPTi uptake [n/N, (\%)] } \\
\hline IPTi (at least one dose) & $3627 / 5035(72)$ & & \\
\hline IPTi (all three doses) & $1133 / 5035(22)$ & & \\
\hline \multicolumn{4}{|l|}{ Median age at IPTi doses [months, (IQR)] } \\
\hline IPTi dose 1 & $2 \mathrm{~m} 15 \mathrm{~d}(2 \mathrm{~m} 3 \mathrm{~d}-3 \mathrm{~m} 6 \mathrm{~d})$ & & \\
\hline IPTi dose 2 & $4 \mathrm{~m} 0 \mathrm{~d}(3 \mathrm{~m} 9 \mathrm{~d}-5 \mathrm{~m} 0 \mathrm{~d})$ & & \\
\hline IPTi dose 3 & $9 m 18 d(9 m 3 d-10 m 12 d)$ & & \\
\hline
\end{tabular}

* Using unpaired t test $\mathrm{p}$ value and approximate $95 \% \mathrm{Cl}$ that adjust for clustering by health centre.

$\mathrm{EPI}=$ expanded programme on immunization, $\mathrm{IQR}=$ inter-quartile range, $\mathrm{m}=$ months, $\mathrm{d}=$ days.

areas for this study were comparable. The total catchment population was 68,938 in comparison areas, compared to 69,042 in IPTi-intervention areas. Analysis of routine health centre data suggested that overall malaria incidence was similar in intervention (1.05 episodes per child per year) and comparison areas (1.10 episodes per child per year), and measles vaccination coverage was also similar (1,647 doses in comparison versus 1,594 doses in intervention areas). Implementation of the Morbidity Monitoring was staggered, beginning in 2005, but a period of maximal functionality was defined as the 12 months between April
2006 and March 2007, and results in this paper refer to this period.

Routine HMIS data, including demographic information, Outpatient department attendance (OPD) and vaccination records were captured electronically by study-trained local school leavers, using Personal Digital Assistants (PDAs) (Palm m130) [25], and records were linked using an identification code. Each PDA operator was working full-time in one of the 11 health centres. They were supported by the study's data entry clerk who visited each health centre monthly. 
In addition to routine HMIS data, blood slide readings for malaria parasites and haemoglobin data were also collected. Malaria parasite blood slides were prepared using Giemsa staining, and parasite counts per 200 white blood cells (WBCs) were calculated [26,27], while haemoglobin was measured using the Haemoglobin Colour Scale [28]. Following reading at the health centre, malaria parasite blood slides were stored and read twice in the Morbidity Monitoring study's quality assurance (QA) laboratory by two independent slide readers [26,29]. Malaria in this study was defined as a health centre attendance which resulted in both a recorded malaria diagnosis by the health centre clinician, and a positive blood slide reading ( $\geq 1$ parasite/200 WBCs) from the QA laboratory. Health centre blood slide results were not used to define malaria, as these had an average sensitivity of $89.6 \%$ and specificity of $77.3 \%$, using the quality assurance laboratory as the gold standard, and variability between health centres was seen. Anaemia was defined using two thresholds; mild anaemia was a haemoglobin $(\mathrm{Hb})$ concentration below $11 \mathrm{~g} / \mathrm{dL}$, and severe anaemia used a threshold of $\mathrm{Hb}<8 \mathrm{~g} / \mathrm{dL}$.

\section{Statistical methods}

Data from PDAs were exported using Pendragon Forms software to Microsoft Access for data management, and subsequently to Stata 10.0 for statistical analysis (Stata Corp., College Station, TX, USA). Analysis was done according to a pre-agreed analytical plan, incorporating both 'intention to treat' and 'per protocol' analyses. In 'intention to treat' analyses, all children attending a sentinel health centre located in divisions randomized to receive IPTi were compared with those attending sentinel health centres located in IPTi comparison divisions. For the 'per protocol' analyses, children attending health centres in intervention areas who had received IPTi were compared with those attending health centres in comparison areas who had received the corresponding EPI vaccines.

Time at risk for malaria, anaemia, and OPD incidence rate calculations began at the latest of the following: date of registration in the consolidated HMIS, date of becoming two months old, or start date of the study $\left(1^{\text {st }}\right.$ April 2006), and ended at the earliest of either becoming 12 months of age, or the end date of the study $\left(31^{\text {st }}\right.$ March 2007). In order to avoid double-counting the same malaria episode as two separate episodes, 28 days follow up was removed after each malaria diagnosis in analyses of multiple episodes of malaria. Incidence rates were calculated using Poisson regression, and incidence rates of multiple episodes of malaria or all-cause health centre attendance were adjusted for within-child clustering using random effects Poisson regression modelling. The protective efficacy of IPTi was defined as $(1-R R) \times 100$, where RR is the rate ratio. All statistical testing was based on the t test, using a summary measure of the data from each health centre, to take into consideration the clustered nature of the health centre data [30].

In addition to analyses specified in the pre-agreed analytical plan, prevalence of malaria and anaemia in infants using data from the 2004 and 2006 IPTi community effectiveness household surveys was also calculated. These surveys are described in detail elsewhere [20,22], but were large, representative of households in the 24 divisions included in the IPTi cluster randomized controlled trial, and the 2006 survey took place in July and August, overlapping the time period of this study. Results of these secondary analyses presented here are restricted to the 11 divisions that were also included in the IPTi Morbidity Monitoring study. Statistical testing was based on cluster level t tests, and analyses were carried out using svy commands in Stata, which are methods appropriate for the clustered design of the IPTi trial and the sampling strategy of the household surveys [30].

\section{Ethics}

The study was undertaken within the framework of the assessment of the community effectiveness of IPTi, part of the IPTi Consortium. Ethical approval was granted by the following local and national institutional review boards: Ifakara Health Institute, National Tanzania Medical Research Coordinating Committee, London School of Hygiene \& Tropical Medicine, and the Cantons of Basel-Stadt and Basel-Land, Switzerland. The permission to execute the project was sought and obtained from the Tanzania Ministry of Health and Social Welfare and the respective District Councils of the participating districts.

\section{Results}

\section{Characteristics of the Morbidity Monitoring study population}

A total of 9,880 children aged 2-11 months contributed at least one day to time at risk between April 2006 and March 2007. Half were from health centres in intervention areas, although some health centres saw more children than others (Table 1). Median registration date was $27^{\text {th }}$ April 2006, and median age at entry into the analysis (four months) was similar in intervention and comparison areas (Table 1). In total, therefore, 7,056 health centre visits were recorded in 4,227 children, with a median of two all-cause health centre attendances per child in both intervention and comparison areas (Table 1).

\section{Uptake of EPI vaccinations and IPTi doses}

During the study, uptake of routine EPI vaccinations was $69 \%$ for DPT2 (children aged 2-11 months), 55\% for DPT3 (children aged 3-11 months), and 42\% for 
measles (children aged 9-11 months). Similar proportions of children from both areas received DPT2; while more children from the intervention areas received DPT3 and measles vaccines (Table 1). Median age at DPT2, DPT3 and measles was 2 months 21 days, 4 months 0 days, and 9 months 18 days respectively, and median age at these routine vaccinations was similar between children in intervention and comparison areas (Table 1). A total of $72 \%$ of children aged 2-11 months in intervention areas received at least one dose of IPTi, and $22 \%$ received all three doses. Median age at IPTi1, IPTi2 and IPTi3 was 2 months 15 days, 4 months 0 days, and 9 months 18 days respectively (Table 1 ).

IPTi and malaria, anaemia, and all-cause OPD attendance Point estimates suggest a protective effect of IPTi on malaria, but an increase in the risk of anaemia, and no difference in the incidence of all-cause outpatient attendance. However, adjustment for the clustered nature of the data means that none of these results reached statistical significance (Tables 2, 3, and 4).

\section{Malaria}

In total during this 12 month period, 763 first or only episodes and 836 multiple episodes of malaria were seen (Table 2). Rates of malaria in IPTi intervention areas appeared lower in 'intention to treat' analysis comparing children attending health centres in IPTi intervention and comparison areas, although differences did not reach statistical significance $(R R=0.75,95 \%$ CI 0.24 2.31 first or only episode of malaria; $\mathrm{RR}=0.82,95 \% \mathrm{CI}$ 0.29-2.29 multiple episodes). In 'per protocol' analyses, comparing children from intervention areas who received IPTi dose 1 with children from comparison

Table 2 Incidence of first or only and all episodes of malaria, and incidence in all time at risk after IPTi dose, and within 28 days of IPTi dose one

\begin{tabular}{|c|c|c|c|c|c|c|c|c|c|}
\hline \multirow[b]{2}{*}{ Intention to treat* } & \multicolumn{3}{|c|}{ Intervention Area } & \multicolumn{3}{|c|}{ Comparison Area } & \multicolumn{3}{|c|}{ Effect } \\
\hline & Events & PYAR & Rate & Events & PYAR & Rate & RR $(95 \% \mathrm{Cl})$ & $p$ value & PE $(95 \% \mathrm{Cl})$ \\
\hline \multicolumn{10}{|c|}{ Malaria incidence (first or only) } \\
\hline Crude & 315 & 2048.3 & 0.154 & 448 & 1882.7 & 0.238 & $\begin{array}{r}0.65(0.56- \\
0.75)\end{array}$ & $<0.001$ & $35 \%(25 \%, 44 \%)$ \\
\hline adjusted $^{\ddagger}$ & & & & & & & $\begin{array}{r}0.75(0.24- \\
2.31)\end{array}$ & 0.584 & $\begin{array}{r}25 \% \\
(-131 \%, 76 \%)\end{array}$ \\
\hline \multicolumn{10}{|c|}{ Malaria incidence (all episodes) } \\
\hline Crude & 329 & 2105.9 & 0.156 & 507 & 1946.0 & 0.261 & $\begin{array}{r}0.60(0.52- \\
0.69)\end{array}$ & $<0.001$ & $40 \%(31 \%, 48 \%)$ \\
\hline \multirow[t]{2}{*}{ adjusted $^{\dagger+}$} & & & & & & & $\begin{array}{r}0.82(0.29- \\
2.29)\end{array}$ & 0.663 & $\begin{array}{r}18 \% \\
(-129 \%, 71 \%)\end{array}$ \\
\hline & IPTi Inte & erventior & Area & $\begin{array}{l}\text { Corres } \\
\text { vaccine }\end{array}$ & $\begin{array}{l}\text { ponding } \\
\text { Compa } \\
\text { Area }\end{array}$ & $\begin{array}{l}g \text { EPI } \\
\text { arison }\end{array}$ & & Effect & \\
\hline Per protocol** & Events & PYAR & Rate & Events & PYAR & Rate & RR $(95 \% \mathrm{Cl})$ & $p$ value & PE $(95 \% \mathrm{Cl})$ \\
\hline \multicolumn{10}{|c|}{$\begin{array}{l}\text { Malaria incidence (all episodes) in ATAR since IPTi } \\
\text { dose }\end{array}$} \\
\hline \multicolumn{10}{|l|}{ IPTi dose 1} \\
\hline crude & 79 & 623.2 & 0.127 & 125 & 513.1 & 0.244 & $\begin{array}{r}0.52(0.39- \\
0.69)\end{array}$ & $<0.001$ & $48 \%(31 \%, 61 \%)$ \\
\hline adjusted $^{\dagger \neq}$ & & & & & & & $\begin{array}{r}0.68(0.21- \\
2.22)\end{array}$ & 0.498 & $\begin{array}{r}32 \% \\
(-122 \%, 79 \%)\end{array}$ \\
\hline \multicolumn{10}{|c|}{ Malaria incidence within 28 days of IPTi dose } \\
\hline crude & 7 & 95.1 & 0.070 & 13 & 81.0 & 0.160 & $\begin{array}{r}0.46(0.18- \\
1.15)\end{array}$ & 0.088 & $54 \%(-15 \%, 82 \%)$ \\
\hline adjusted $^{\ddagger}$ & & & & & & & $\begin{array}{r}0.48(0.11- \\
2.13)\end{array}$ & 0.341 & $\begin{array}{r}52 \% \\
(-113 \%, 89 \%)\end{array}$ \\
\hline
\end{tabular}

*Intention to treat analyses comparing IPTi intervention and comparison areas.

**Per protocol analyses comparing children who received IPTi in intervention areas to those that received corresponding EPI vaccines in comparison areas. $\mathrm{EPI}=$ expanded programme on immunization, $\mathrm{PAYR}=$ person years at risk, $\mathrm{ATAR}=$ all time at risk, $\mathrm{RR}=$ rate ratio, $\mathrm{Cl}=\mathrm{confidence}$ interval, $\mathrm{PE}=\mathrm{protective}$ efficacy.

${ }^{\dagger}$ Results adjusted for clustering within children for multiple episodes of malaria using Poisson random effects modeling.

*Using intervention and comparison area mean rate, unpaired t test $\mathrm{p}$ value and approximate $95 \% \mathrm{Cl}$ that adjust for clustering by health centre. 
Table 3 Incidence of first or only episode of mild and severe anaemia, and incidence of anaemia in all time at risk, and within 28 days of IPTi dose one

\begin{tabular}{|c|c|c|c|c|c|c|c|c|c|}
\hline \multirow[b]{2}{*}{ Intention to treat* } & \multicolumn{3}{|c|}{ Intervention Area } & \multicolumn{3}{|c|}{ Comparison Area } & \multicolumn{3}{|c|}{ Effect } \\
\hline & Events & PYAR & Rate & Events & PYAR & Rate & RR $(95 \% \mathrm{Cl})$ & $p$ value & PE $(95 \% \mathrm{Cl})$ \\
\hline \multicolumn{10}{|c|}{ Mild anaemia incidence (first or only) } \\
\hline Crude & 1268 & 1771.8 & 0.716 & 1170 & 1689.7 & 0.694 & $1.03(0.95-1.12)$ & 0.424 & $-3 \%(-12 \%, 5 \%$ \\
\hline adjusted $^{\ddagger}$ & & & & & & & $1.13(0.65-1.97)$ & 0.597 & $-13 \%(-97 \%, 35 \%)$ \\
\hline \multicolumn{10}{|c|}{ Severe anaemia incidence (first or only) } \\
\hline crude & 318 & 2046.8 & 0.155 & 206 & 1944.1 & 0.106 & $1.47(1.23-1.75)$ & $<0.001$ & $-47 \%(-75 \%,-23 \%)$ \\
\hline \multirow[t]{2}{*}{ adjusted $^{\ddagger}$} & & & & & & & $1.59(0.54-4.68)$ & 0.360 & $-59 \%(-368 \%, 46 \%)$ \\
\hline & IPTi Inte & erventior & n Area & $\begin{array}{l}\text { Corres } \\
\text { vaccine }\end{array}$ & $\begin{array}{l}\text { sponding } \\
\text { e Compa } \\
\text { Area }\end{array}$ & $\begin{array}{l}\text { g EPI } \\
\text { arison }\end{array}$ & & Effect & \\
\hline Per protocol ${ }^{* *}$ & Events & PYAR & Rate & Events & PYAR & Rate & RR $(95 \% \mathrm{Cl})$ & $p$ value & PE $(95 \% \mathrm{Cl})$ \\
\hline \multicolumn{10}{|c|}{$\begin{array}{l}\text { Mild anaemia incidence (first or only) in ATAR } \\
\text { since IPTi dose }\end{array}$} \\
\hline \multicolumn{10}{|c|}{ IPTi dose 1} \\
\hline crude & 289 & 543.8 & 0.531 & 257 & 455.6 & 0.564 & $0.93(0.78-1.10)$ & 0.383 & $7 \%(-10 \%, 22 \%)$ \\
\hline adjusted $^{\ddagger}$ & & & & & & & $1.04(0.58-1.87)$ & 0.878 & $-4 \%(-87 \%, 42 \%)$ \\
\hline \multicolumn{10}{|c|}{$\begin{array}{l}\text { Severe anaemia incidence (first or only) in ATAR } \\
\text { since IPTi dose }\end{array}$} \\
\hline \multicolumn{10}{|c|}{ IPTi dose 1} \\
\hline crude & 65 & 615.2 & 0.106 & 26 & 518.3 & 0.050 & $2.10(1.34-3.32)$ & 0.001 & $\begin{array}{r}-110 \%(-232 \%,- \\
34 \%)\end{array}$ \\
\hline adjusted $^{\ddagger}$ & & & & & & & $2.12(0.41-11.03)$ & 0.277 & $\begin{array}{r}-112 \% \\
(-1000 \%, 59 \%)\end{array}$ \\
\hline
\end{tabular}

Mild anaemia incidence (first or only) within 28 days of IPTi dose

IPTi dose 1

\begin{tabular}{|c|c|c|c|c|c|c|c|c|c|}
\hline crude & 45 & 93.8 & 0.480 & 31 & 80.5 & 0.385 & $1.25(0.79-1.97)$ & 0.343 & $-25 \%(-97 \%, 21 \%)$ \\
\hline adjusted $^{\ddagger}$ & & & & & & & $1.30(0.42-4.07)$ & 0.579 & $-30 \%(-307 \%, 58 \%)$ \\
\hline
\end{tabular}

Severe anaemia incidence (first or only) within 28

days of IPTi dose

IPTi dose 1

\begin{tabular}{lllllllllll}
$\begin{array}{l}\text { crude } \\
\text { adjusted }^{\ddagger}\end{array}$ & 4 & 95.1 & 0.042 & 2 & 81.4 & 0.025 & 1.71 & $(0.31-9.35)$ & 0.530 & $-71 \%(-835 \%, 69 \%)$ \\
1.57 & $(0.11-22.93)$ & 0.700 & $-57 \%(-2190 \%, 89 \%)$ \\
\hline
\end{tabular}

*Intention to treat analyses comparing IPTi intervention and comparison areas.

**Per protocol analyses comparing children who received IPTi in intervention areas to those that received corresponding EPI vaccines in comparison areas.

$\mathrm{EPI}=$ expanded programme on immunization, PAYR = person years at risk, $\mathrm{ATAR}=$ all time at risk, $\mathrm{RR}=$ rate ratio,

$\mathrm{Cl}=$ confidence interval, $\mathrm{PE}=$ protective efficacy,

$\mathrm{Hb}=$ haemoglobin.

${ }^{\ddagger}$ Using intervention and comparison area mean rate, unpaired t test $\mathrm{p}$ value and approximate $95 \% \mathrm{Cl}$ that adjust for clustering by health centre.

areas who received DPT2, 204 episodes of malaria were seen in all time at risk after receiving IPTi dose 1 , and 20 episodes were recorded within 28 days of IPTi dose 1. Rates of malaria in children who received IPTi 1 appeared lower, but differences were not statistically significant $(R R=0.68,95 \%$ CI $0.21-2.22$ for episodes in all time at risk after IPTi 1 ; RR $=0.48,95 \%$ CI $0.11-2.13$ for episodes with 28 days). Protective efficacy point estimates in both 'intention to treat' and 'per protocol' analyses were consistently lower on adjustment for clustering by health centre.

\section{Anaemia}

Table 3 shows that 2438 episodes of mild anaemia $(\mathrm{Hb}$ $<11 \mathrm{~g} / \mathrm{dL})$, and 524 episodes of severe anaemia $(\mathrm{Hb}<8$ $\mathrm{g} / \mathrm{dL}$ ) were recorded during the 12 month study period. Point estimates of protective efficacy in 'intention to treat' analyses suggest rates in children attending health centres in IPTi intervention areas were higher that those attending centres in comparison areas, although differences were not statistically significant $(R R=1.13,95 \%$ CI 0.65-1.97 mild anaemia; $R R=1.59,95 \%$ CI 0.54-4.68 severe anaemia). In 'per protocol' analyses 546 episodes 
Table 4 Incidence of first or only and all OPD attendances, and incidence of attendance in all time at risk, and within 28 days of IPTi dose one

\begin{tabular}{|c|c|c|c|c|c|c|c|c|c|}
\hline \multirow[b]{2}{*}{ Intention to treat* } & \multicolumn{3}{|c|}{ Intervention Area } & \multicolumn{3}{|c|}{ Comparison Area } & \multicolumn{3}{|c|}{ Effect } \\
\hline & Events & PYAR & Rate & Events & PYAR & Rate & RR $(95 \% \mathrm{Cl})$ & $\begin{array}{c}p \\
\text { value }\end{array}$ & PE $(95 \% \mathrm{Cl})$ \\
\hline \multicolumn{10}{|c|}{ Health centre attendance (first or only) } \\
\hline crude & 2160 & 1535.3 & 1.407 & 2067 & 1434.4 & 1.441 & $0.97(0.92-1.04)$ & 0.417 & $3 \%(-4 \%, 8 \%)$ \\
\hline adjusted $^{\ddagger}$ & & & & & & & $1.04(0.75-1.45)$ & 0.795 & $-4 \%(-45 \%, 25 \%)$ \\
\hline \multicolumn{10}{|c|}{ Health centre attendances (all episodes) } \\
\hline crude & 3631 & 1810.5 & 2.005 & 3425 & 1699.3 & 2.015 & $0.99(0.95-1.04)$ & 0.834 & $1 \%(-4 \%, 5 \%)$ \\
\hline \multirow[t]{2}{*}{ adjusted $^{\dagger \neq}$} & & & & & & & $1.05(0.75-1.48)$ & 0.736 & $-5 \%(-48 \%, 25 \%)$ \\
\hline & IPTi Inte & erventior & n Area & $\begin{array}{l}\text { Corres } \\
\text { vaccine }\end{array}$ & $\begin{array}{l}\text { ponding } \\
\text { e Compa } \\
\text { Area }\end{array}$ & $\begin{array}{l}\text { g EPI } \\
\text { arison }\end{array}$ & & Effect & \\
\hline Per protocol** & Events & PYAR & Rate & Events & PYAR & Rate & RR $(95 \% \mathrm{Cl})$ & $\begin{array}{c}p \\
\text { value }\end{array}$ & PE (95\% Cl) \\
\hline
\end{tabular}

Health centre attendances (all episodes) in ATAR since

IPTi dose

IPTi dose 1

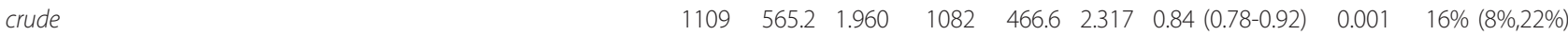

adjusted $^{\dagger} \neq$

$0.98(0.69-1.38) \quad 0.907 \quad 2 \%(-38 \%, 31 \%)$

Health centre attendance (all episodes) within 28 days of IPTi dose

IPTi dose 1

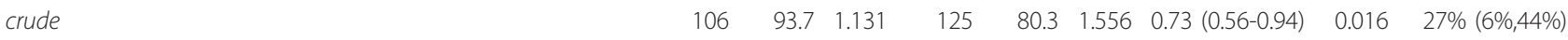

\begin{tabular}{lrrr} 
adjusted $^{\dagger \neq}$ & $0.82(0.48-1.40)$ & 0.421 & $18 \%$ \\
\hline
\end{tabular}

*Intention to treat analyses comparing IPTi intervention and comparison areas.

**Per protocol analyses comparing children who received IPTi in intervention areas to those that received corresponding EPI vaccines in comparison areas. $\mathrm{EPI}=$ expanded programme on immunization, $\mathrm{PAYR}=$ person years at risk, $\mathrm{ATAR}=$ all time at risk, $\mathrm{RR}=$ rate ratio, $\mathrm{Cl}=$ confidence interval, $\mathrm{PE}=$ protective efficacy.

${ }^{\dagger}$ Results adjusted for clustering within children for multiple episodes of all-cause health centre attendance using Poisson random effects modeling.

${ }^{*}$ Using intervention and comparison area mean rate, unpaired $t$ test $p$ value and approximate $95 \% \mathrm{Cl}$ that adjust for clustering by health centre.

of mild anaemia were seen in all time at risk (ATAR) since IPTi dose 1 , and 76 episodes were recorded within 28 days. Rates of severe anaemia were much lower, with 91 in all time at risk after IPTi dose 1, and only six cases of severe anaemia within 28 days of IPTi dose 1. Rates of mild and severe anaemia from 'per protocol' analyses were also higher in children who had received IPTi dose 1, in comparison to those who had received DPT2, but results were not statistically significant (mild anaemia: $\mathrm{RR}=1.04,95 \% \mathrm{CI}$ 0.58-1.87 $\mathrm{ATAR}$ and $\mathrm{RR}=$ 1.30, $95 \%$ CI $0.42-4.07$ within 28 days; severe anaemia: $\mathrm{RR}=2.12,95 \%$ CI $0.41-11.03 \mathrm{ATAR}$, and $\mathrm{RR}=1.57$, 95\% CI (0.11-22.93) within 28 days).

\section{All-cause attendance at the health centre}

Over the 12 months from April 2006 to March 2007, 4,227 first or only attendances, and 7,056 multiple attendances at the health centre were seen (Table 4). Rates of attendance in children visiting health centres in IPTi intervention areas were similar to those in children attending centres in comparison areas. In 'per protocol' analyses 2191 attendances were recorded in all time at risk after IPTi dose 1, and 231 attendances were recorded within 28 days of IPTi 1 . Rates of all-cause health centre attendance were similar in children from IPTi intervention areas who received IPTi dose 1 than in those from comparison areas who had received DPT2.

\section{Malaria and anaemia prevalence in the 2004 and 2006 IPTi household surveys, restricted to Morbidity Monitoring divisions}

When restricted to the 11 divisions included in the Morbidity Monitoring study, information was available from 257 and 319 children aged 2-11 months in the 2004 and 2006 household surveys respectively. Results from the 2004 survey (prior to IPTi), suggest that the 11 divisions included in the IPTi Morbidity Monitoring study were comparable on variables for which information was available (Table 5). Changes between 2004 and 2006 in the prevalence of parasitaemia, severe anaemia and net use by children in the night before the survey (Figure 2, Table 5), show a marked decrease in malaria 
Table 5 Prevalence of parasitaemia, net use and mean haemoglobin levels in children aged 2-11 months, using data from the 2004 and 2006 IPTi Household Surveys, restricted to Morbidity Monitoring divisions $(n=11)$

2004 IPTi household survey Intervention Comparison $\mathrm{p}$ value *

\begin{tabular}{llll}
\hline Parasitaemia [n/N (\%)] & 98/156 (63) & $60 / 101(59)$ & 0.677
\end{tabular}

Haemoglobin $(\mathrm{g} / \mathrm{dL})[$ mean $\quad 8.5(8.3-8.8) \quad 8.8(8.5-9.1) \quad 0.955$ (95\% Cl)]

Net use (last night) [n/N (\%)] $\quad$ 45/156 (29) $\quad$ 27/101 (27) 0.739

2006 IPTi household survey Intervention Comparison $\mathrm{p}$ value *

\begin{tabular}{llll}
\hline Parasitaemia [n/N (\%)] & $62 / 188(33)$ & $47 / 111(42)$ & 0.130
\end{tabular}

Haemoglobin $(\mathrm{g} / \mathrm{dL})[$ mean $\quad 9.6(9.3-9.9) \quad 9.3(8.9-9.6) \quad 0.640$ (95\% Cl)]

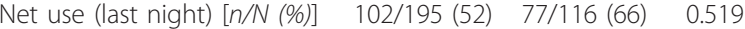

Results obtained using svy commands in Stata, which adjust for the sampling strategy of the survey.

$\mathrm{Hb}=$ haemoglobin, $\mathrm{Cl}=$ confidence interval.

*Using unpaired $t$ test that adjusts for the IPTi trial study design (randomized by division). parasitaemia, and an increase in mean haemoglobin and bed net usage in both IPTi intervention and comparison areas. In 2006, in Morbidity Monitoring areas, differences between intervention and comparison areas did not reach statistical significance, but prevalence of parasitaemia was nine percentage points lower in intervention areas, while mean haemoglobin was $0.3 \mathrm{~g} / \mathrm{dL}$ higher in intervention areas, and prevalence of reported bed net use by children in the night before the survey was 14 percentage points higher in comparison areas (Table 5).

\section{Discussion}

\section{Effectiveness of IPTi with SP}

In all, $72 \%$ of children aged $2-11$ months attending health centres in intervention areas received at least one dose of IPTi, and $22 \%$ received all three doses. Median age at IPTi dose was older than recommended by EPI guidelines, but similar to median ages at routine

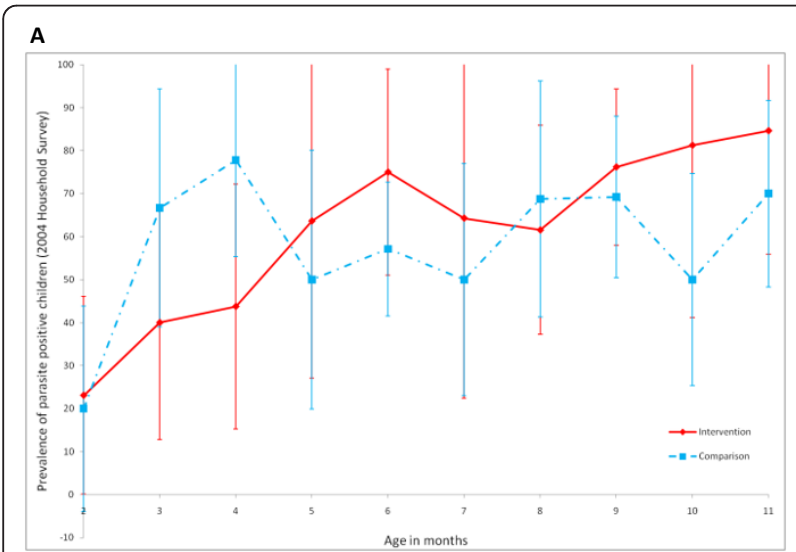

C

B
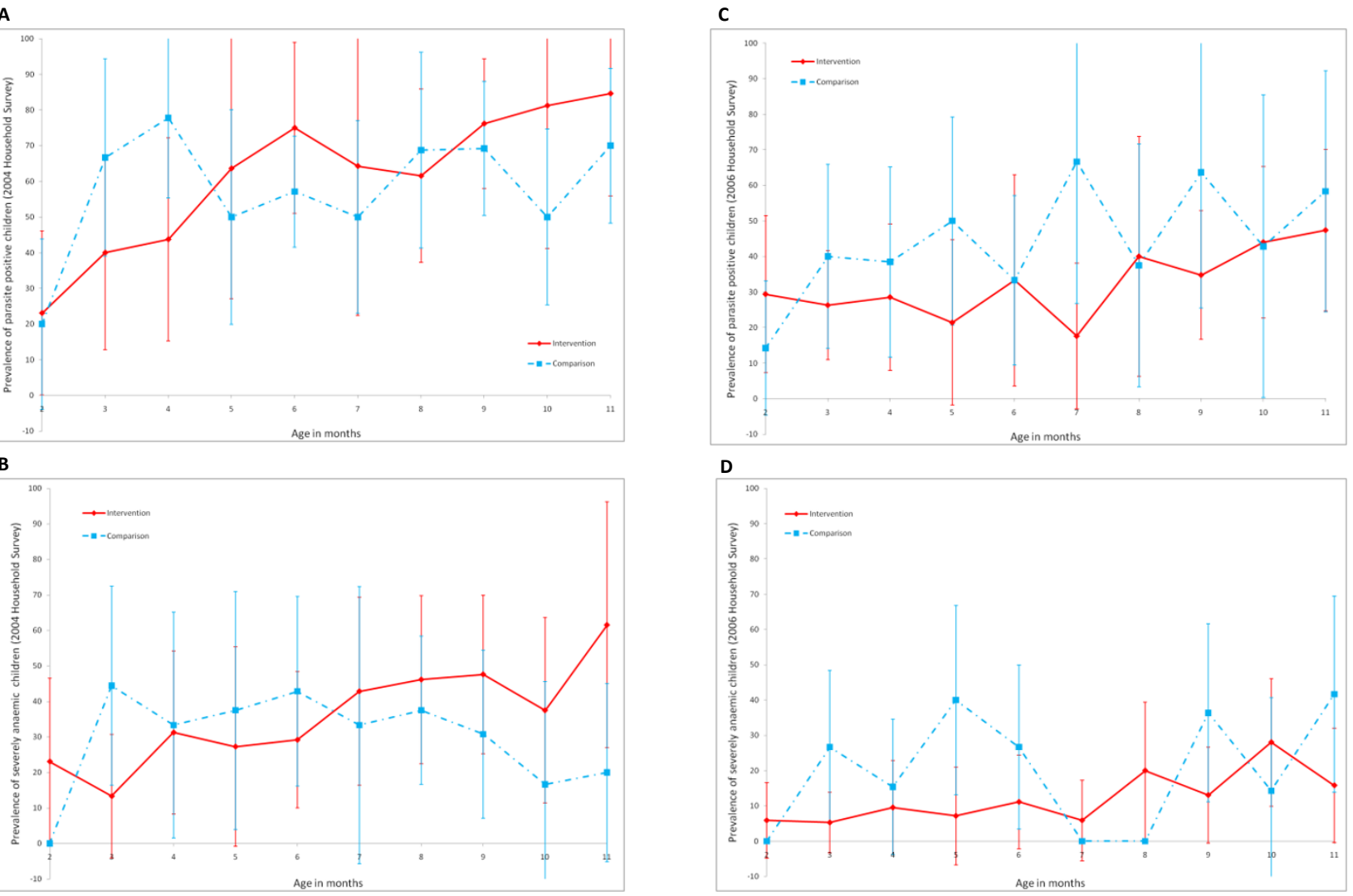

Figure 2 Prevalence of parasitaemia and severe anaemia $(\mathrm{Hb}<8 \mathrm{~g} / \mathrm{dL})$ with $95 \%$ confidence intervals, by age in months, in children aged 2-11 months in Morbidity Monitoring divisions $(n=11)$. Data from 2004 and 2006 IPTi Household Surveys. (A) Prevalence of parasite positive children (2004 Household Survey), $n=257$. (B) Prevalence of severely anaemic children (2004 Household Survey), $n=257$. (C) Prevalence of parasite positive children (2006 Household Survey), $n=$ 299. (D) Prevalence of severely anaemic children (2006 Household Survey), $\mathrm{n}=299 . \mathrm{Hb}=$ haemoglobin. 
vaccinations, and similar to those seen during the 2006 IPTi representative household survey [22]. Results suggest that more children from intervention areas received DPT2 and measles vaccine, however, results from the representative 2006 IPTi household survey, carried out during a time period that overlapped this study, show no indication of increased levels of EPI coverage in IPTi intervention areas-coverage of DPT vaccination was identical in both areas at $74 \%$ of children aged 6-11 months [22].

Overall incidence rates of 0.2 episodes of lab-confirmed malaria; 0.7 episodes of mild and 0.13 of severe anaemia; and 2 episodes of all-cause health centre attendance per child per year were seen. These analyses of routine health information data suggest that IPTi was associated with an increased risk of anaemia, a reduced risk of malaria, an increase in coverage of routine vaccinations, and no effect on all cause out-patient attendance. However, none of the effects of IPTi on malaria, anaemia or all-cause health centre attendance, in either 'intention to treat' or 'per protocol' analyses, reached statistical significance when adjusted for clustering by health centre. The confidence intervals for the rate ratios of the effect of IPTi with SP on these outcomes included the null value of one, and ranged from rate ratios that are compatible with a protective effect of IPTi, to rate ratios that suggest increased rates of these outcomes in the IPTi group. These results could represent (i) that IPTi with SP has no impact on malaria, anaemia or all-cause attendance at the health centre in children aged 2-11 months in this setting, (ii) there was insufficient statistical power to detect an effect of IPTi with SP, and linked to this, (iii) that the background rates of malaria and malaria-related anaemia were too low, due possibly to other control measures including net use, to detect an impact of IPTi with the existing study design.

Studies, including a pooled analysis of six large RCTs of IPTi using SP [5], have reported an IPTi-related reduction in malaria and health centre attendance in infants. Increased rates of anaemia have been reported in two IPTi trials $[9,11]$, however, the majority of RCTs of IPTi have reported a decline $[7,8,12]$, or no effect [10], with results of the pooled analysis showing a $22 \%$ decline in severe anaemia $(\mathrm{Hb}<8 \mathrm{~g} / \mathrm{dL})$ [5]. Results from the 2006 IPTi household survey carried out in July and August, and including all 24 divisions, indicated that prevalence of mild anaemia $(\mathrm{Hb}<11 \mathrm{~g} / \mathrm{dL})$ was $8 \%$ lower $(\mathrm{p}=0.02)$ in children from IPTi intervention areas, while in children who had received IPTi, mean haemoglobin was 0.27 grams higher $(p=0.05)$ than in children who had received the corresponding EPI vaccine [22]. Given findings from the wider literature, it is suggested that the discrepancy in the results seen in this study, and those from the IPTi household survey carried out during an overlapping time period in the same geographical area, is more likely due to the non-representative nature of infants attending health facilities in comparison to the whole population of infants, rather than to a true increased risk in anaemia associated with IPTi using SP.

All health centres with functional microscopes in our project area were included in this evaluation $(n=11)$. However, this provided only $32 \%$ power to detect a $30 \%$ difference between IPTi intervention and comparison areas. An additional 19 centres (30 in total) would be required to achieve $80 \%$ power [30], although this would not have been feasible, as in reality all eligible centres in participating divisions were included.

In the 11 divisions included in the Morbidity Monitoring study, there was a $17 \%$ fall in malaria parasitaemia between 2004 and 2006 in children aged 2-11 months of age living in IPTi comparison areas, as measured by the representative household surveys. Using the same source data, it was shown that in children of this age group in IPTi comparison areas there was also a $0.5 \mathrm{~g} / \mathrm{dL}$ increase in mean haemoglobin, and a $39 \%$ increase in the reported use of nets by children on the night before the survey. These background changes may also have contributed to the current study lacking sufficient power to detect an effect of IPTi with SP. Longer periods of observation may enable the discernment of trends, and a difference between these trends in intervention and comparison areas. These observations also draw attention to the need to monitor contextual factors which may impact on the evaluation.

\section{The use of routine HMIS data to monitor and evaluate malaria control programmes}

This study used HMIS data to evaluate the effectiveness of IPTi with SP on health centre attendees. Findings may, therefore, not be representative of children, and their morbidity burden, in the wider community [31].

The quality of data in HMIS remains a critical issue. Monthly supervision visits to all study sites were carried out by the study co-coordinator (WM), data manager (KS), and laboratory assistant. Visits were an opportunity to discuss issues of data completeness and quality with health centre staff. The basis for these discussions was an automatically-, PDA-generated monthly report. These compared numbers of OPD attendances and EPI vaccinations to those recorded during the previous month; highlighted whether all OPD attendances had corresponding laboratory records; and listed the top ten diagnoses made at the centre in the past month, which previously had been prepared manually. Use of PDAs enhanced data capture, ease of retrieval and summarising of the information. However, despite monthly supervision, issues of data completeness across HMIS 
registers, and the quality of microscopy from the on-site health centre laboratories remained.

For example, on average $41 \%$ of blood slide results from the health centre laboratory were missing a corresponding record in the OPD register, and this showed variability between the different health centres. This study used blood slide results from the Morbidity Monitoring quality assurance laboratory, along with clinical diagnosis from HMIS, to classify malaria outcome. Health centre microscopy results had an average sensitivity of $89.6 \%$ and specificity of $77.3 \%$, using the QA lab as gold standard. This again showed variation between health centres. There was no suggestion that data completeness or quality of microscopy was consistently better or worse in IPTi intervention or comparison areas.

The use of HMIS data for monitoring and evaluation should undergo quality control checks, be analysed with due regard to potential selection biases (e.g. the type of patient attending different health facilities), clustering of the data, and statistical power considerations. The data need to be interpreted bearing in mind the contextual situation and consistency of results. Where unexpected findings are documented, well-designed, well-implemented, representative household surveys may be needed to answer the question of intervention effectiveness at the community level, also capturing data from those populations who do not attend routine health services.

\section{Conclusions}

This study used routine data from Health Management Information Systems in 11 sentinel health centres in rural southern Tanzania to evaluate the effectiveness of a new malaria control strategy, IPTi with SP. The process highlights the challenges of using routine health centre data to monitor and evaluate the effectiveness of a malaria control programme in health centre attendees. Findings suggest that unexpected results should be investigated by drawing representative samples of the target population to enable robust conclusions to be drawn.

\section{Acknowledgements \\ We would like to thank health staff in the sentinel health centres, PDA operators, and Regional and District Health Management Teams of the participating regions and districts. We appreciate the cooperation offered by the Tanzania Ministry of Health and Social Welfare, and the administrative support given by the Ifakara Health Institute. This study was financially supported by the Bill and Melinda Gates Foundation through the IPTi consortium. JAS was supported by funding from Gates Malaria Partnership. JAS and BW are members of the MRC Tropical Epidemiology Group at the London School of Hygiene \& Tropical Medicine.}

\section{Author details}

${ }^{1}$ Faculty of Infectious and Tropical Diseases, London School of Hygiene and Tropical Medicine, London, UK. 'Ifakara Health Institute, Dar es Salaam, Tanzania. ${ }^{3}$ Barcelona Centre for International Health Research, Barcelona,
Spain. ${ }^{4}$ Swiss Tropical and Public Health Institute, Basel, Switzerland. ${ }^{5}$ University of Basel, Basel, Switzerland.

\section{Authors' contributions}

The paper was drafted by BW, WM, JAS and DS. BW carried out the statistical analyses, with support from JAS. DS, JAS, HM, PA and MT participated in the set up and management of the Morbidity Monitoring study. WM, KS and MC were responsible for data collection. All authors commented on drafts of the paper and approved the final version.

\section{Competing interests}

The authors declare that they have no competing interests.

Received: 28 September 2010 Accepted: 14 February 2011 Published: 14 February 2011

\section{References}

1. World Health Organization: World Malaria Report. World Health Organization; 2008.

2. WHO Roll Back Malaria: Framework for Monitoring Progress \& Evaluation Outcomes and Impact. World Health Organization; 2000.

3. WHO Roll Back Malaria Monitoring and Evaluation Reference Group: Building capacity in monitoring and evaluating Roll Back Malaria in Africa: A conceptual framework for the Roll Back Malaria Partnership. WHO Roll Back Malaria Monitoring and Evaluation Reference Group (MERG); 2005

4. Egan A, Crawley J, Schellenberg D: Editorial: Intermittent preventive treatment for malaria control in infants: moving towards evidence-based policy and public health action. Trop Med Int Health 2005, 10:815-817.

5. Aponte JJ, Schellenberg D, Egan A, Breckenridge A, Carneiro I, Critchley J, Danquah I, Dodoo A, Kobbe R, Lell B: Efficacy and safety of intermittent preventive treatment with sulfadoxine-pyrimethamine for malaria in African infants: a pooled analysis of six randomised, placebo-controlled trials. Lancet 2009, 374:1533-1542.

6. Gosling RD, Carneiro I, Chandramohan D: Intermittent preventive treatment of malaria in infants: how does it work and where will it work? Trop Med Int Health 2009, 14:1003-1010.

7. Chandramohan D, Owusu-Agyei S, Carneiro I, Awine T, Amponsa-Achiano K, Mensah N, Jaffar S, Baiden R, Hodgson A, Binka F, Greenwood B: Cluster randomised trial of intermittent preventive treatment for malaria in infants in area of high, seasonal transmission in Ghana. BMJ 2005, 331:727-733.

8. Grobusch MP, Lell B, Schwarz NG, Gabor J, Dornemann J, Potschke M Oyakhirome S, Kiessling GC, Necek M, Langin MU: Intermittent preventive treatment against malaria in infants in Gabon - A randomized, doubleblind, placebo-controlled trial. J Infect Dis 2007, 196:1595-1602.

9. Kobbe R, Kreuzberg C, Adjei S, Thompson B, Langefeld I, Thompson PA, Abruquah HH, Kreuels B, Ayim M, Busch W: A randomized controlled trial of extended intermittent preventive antimalarial treatment in infants. Clin Infect Dis 2007, 45:16-25.

10. Macete E, Aide P, Aponte JJ, Sanz S, Mandomando I, Espasa M, Sigauque B, Dobano C, Mabunda S, DgeDge M: Intermittent preventive treatment for malaria control administered at the time of routine vaccinations in Mozambican infants: A randomized, placebo-controlled trial. J Infect Dis 2006, 194:276-285.

11. Mockenhaupt FP, Reither K, Zanger P, Roepcke F, Danquah I, Saad E, Ziniel P, Dzisi SY, Frempong M, Agana-Nsiire P: Intermittent preventive treatment in infants as a means of malaria control: a randomized, double-blind, placebo-controlled trial in northern Ghana. Antimicrob Agents Chemother 2007, 51:3273-3281.

12. Schellenberg D, Menendez C, Kahigwa E, Aponte J, Vidal J, Tanner M, Mshinda $\mathrm{H}$, Alonso P: Intermittent treatment for malaria and anaemia control at time of routine vaccinations in Tanzanian infants: a randomised, placebo-controlled trial. Lancet 2001, 357:1471-1477.

13. Hutton G, Schellenberg D, Tediosi F, Macete E, Kahigwa E, Sigauque B, Mas X, Trapero M, Tanner M, Trilla A: Cost-effectiveness of malaria intermittent preventive treatment in infants (IPTi) in Mozambique and the United Republic of Tanzania. Bull World Health Organ 2009, 87:123-129.

14. Manzi F, Hutton G, Schellenberg J, Tanner M, Alonso P, Mshinda H, Schellenberg D: From strategy development to routine implementation: 
the cost of Intermittent Preventive Treatment in Infants for malaria control. BMC Health Services Research 2008, 8.

15. Pearce RJ, Pota H, Evehe M-SB, Ba E-H, Mombo-Ngoma G, Malisa AL, Ord R, Inojosa W, Matondo A, Diallo DA, et al: Multiple origins and regional dispersal of resistant dhps in African Plasmodium falciparum malaria. PLOS Med 2009, 6:e1000055.

16. Pool R, Munguambe K, Macete E, Aide P, Juma G, Alonso P, Menendez C: Community response to intermittent preventive treatment delivered to infants (IPTi) through the EPI system in Manhica, Mozambique. Trop Med Int Health 2006, 11:1670-1678.

17. Pool R, Mushi A, Schellenberg JA, Mrisho M, Alonso P, Montgomery C, Tanner M, Mshinda H, Schellenberg D: The acceptability of intermittent preventive treatment of malaria in infants (IPTi) delivered through the expanded programme of immunization in southern Tanzania. Malar $J$ 2008, 7:213.

18. Manzi F, Schellenberg J, Hamis Y, Mushi AK, Shirima K, Mwita A, Simba A, Rusibamayila N, Kitambi M, Tanner M: Intermittent preventive treatment for malaria and anaemia control in Tanzanian infants; the development and implementation of a public health strategy. Trans R Soc Trop Med Hyg 2009, 103:79-86.

19. Schellenberg D, Cisse B, Menendez C: The IPTi Consortium: research for policy and action. Trends Parasitol 2006, 22:296-300.

20. Armstrong Schellenberg JRM, Mrisho M, Manzi F, Shirima K, Mbuya C, Mushi AK, Ketende SC, Alonso PL, Mshinda H, Tanner M, Schellenberg D: Health and survival of young children in southern Tanzania. BMC Public Health 2008, 8:194

21. Armstrong Schellenberg JRMSK, Maokola W, Manzi F, Mrisho M, Mushi A, Mshinda H, Alonso P, Tanner M, Schellenberg D: Community effectiveness of Intermittent Preventive Treatment for infants (IPTi) in rural southern Tanzania. Am J Trop Med Hyg 2010, 82:772-781.

22. Armstrong Schellenberg JRMSK, Maokola W, Manzi F, Mrisho M, Mushi A, Mshinda H, Alonso P, Tanner M, Schellenberg D: Community effectiveness of Intermittent Preventive Treatment for infants (IPTi) in rural southern Tanzania. Am J Trop Med Hyg2010 82:772-81.

23. Hanson K, Marchant T, Nathan R, Mponda H, Jones C, Bruce J, Mshinda H, Armstrong J: Household ownership and use of insecticide treated nets among target groups after implementation of a national voucher programme in the United Republic of Tanzania: plausibility study using three annual cross sectional household surveys. BMJ 2009, 339.

24. Skarbinski J, Massaga JJ, Rowe AK, Kachur SP: Distribution of free untreated bednets bundled with insecticide via an integrated child health campaign in Lindi Region, Tanzania: Lessons for future campaigns. Am J Trop Med Hyg 2007, 76:1100-1106.

25. Shirima K, Mukasa O, Schellenberg J, Manzi F, John D, Mushi A, Mrisho M, Tanner M, Mshinda H, Schellenberg D: The use of personal digital assistants for data entry at the point of collection in a large household survey in southern Tanzania. Emerging Themes in Epidemiology 2007, 4:5.

26. Alonso PL, Smith T, Schellenberg J, Masanja H, Mwankusye S, Urassa H, Deazevedo IB, Chongela J, Kobero S, Menendez C: Randomized trial of efficacy of Spf66 Vaccine against Plasmodium falciparum malaria in children in southern Tanzania. Lancet 1994, 344:1175-1181.

27. O'Meara WP, Remich S, Ogutu B, Lucas M, Mtalib R, Obare P, Oloo F, Onoka C, Osoga J, Ohrt C, McKenzie FE: Systematic comparison of two methods to measure parasite density from malaria blood smears. Parasitol Res 2006, 99:500-504.

28. Gosling R, Walraven G, Manneh F, Bailey B, Lewis SM: Training health workers to assess anaemia with the WHO haemoglobin colour scale. Trop Med Int Health 2000, 5:214-221.

29. O'Meara WP, McKenzie FE, Magill AJ, Forney JR, Permpanich B, Lucas C, Gasser RA, Wongsrichanalai C: Sources of variability in determining malaria parasite density by microscopy. Am J Trop Med Hyg 2005, 73:593-598.

30. Hayes RJ, Moulton LH: Cluster randomised trials. Boca Ranton: Taylor \& Francis; 2009.

31. Rowe A, Kachur SP, Yoon S, Lynch M, Slutsker L, Steketee R: Caution is required when using health facility-based data to evaluate the health impact of malaria control efforts in Africa. Malar J 2009, 8:209.

doi:10.1186/1475-2875-10-41

Cite this article as: Willey et al:: Evaluating the effectiveness of IPTi on malaria using routine health information from sentinel health centres in southern Tanzania. Malaria Journal 2011 10:41.

\section{Submit your next manuscript to BioMed Central and take full advantage of:}

- Convenient online submission

- Thorough peer review

- No space constraints or color figure charges

- Immediate publication on acceptance

- Inclusion in PubMed, CAS, Scopus and Google Scholar

- Research which is freely available for redistribution

Submit your manuscript at www.biomedcentral.com/submit
Biomed Central 